\title{
Herramientas de Simulación para Sistemas Fotovoltaicos en Ingeniería
}

\author{
S. Silvestre ${ }^{1}$, L. Castañer ${ }^{1}$ y D. Guasch ${ }^{2}$ \\ (1) Universidad Politécnica de Cataluña, E.T.S.I.T., Departamento de Ingeniería Electrónica, \\ Calle Gran Capitán s/n, Módulo C-4, Campus Norte, 08034 Barcelona-España \\ (e-mail: santi@eel.upc.edu, castaner@eel.upc.edu) \\ (2) Universidad Politécnica de Cataluña, E.P.S.E.V.G., Departamento de Ingeniería Telemática, \\ Avda. Víctor Balaguer s/n, 08800 Vilanova i la Geltrú-España (e-mail: daniel.guasch@upc.edu)
}

\begin{abstract}
Resumen
Este trabajo presenta la simulación de un sistema fotovoltaico, basado en el entorno abierto de programación Matlab/Simulink, utilizado en la asignatura Sistemas Fotovoltaicos, dictado en un programa de Magíster de la Universidad Politécnica de Cataluña, España. Para su aplicación se han desarrollado modelos de todos los componentes de un sistema fotovoltaico. El método permite un rápido y fácil acceso a la evolución de las corrientes y tensiones del sistema. Este ejemplo de simulación permite a los estudiantes, un acceso cómodo y rápido al análisis y dimensionado de un sistema fotovoltaico, lo que facilita una visión global clara del comportamiento del sistema en distintos entornos de funcionamiento.
\end{abstract}

\section{Simulation tools in Photovoltaic Systems Engineering}

\begin{abstract}
This work shows the simulation of a photovoltaic system, using Matlab/Simulink, applied in a course of Photovoltaic Systems in a Master Program of the Polytechnic University of Cataluña, Spain. Different models for all the components of the photovoltaic system have been developed. This method allows an easy and quick access to the system voltages and currents evolution. This simulation example provides the students a good and easy access to the analysis and design of a photovoltaic system, giving a good idea of the system behaviour under different working conditions.
\end{abstract}

Keywords: photovoltaic systems, simulation, electronics engineering, teaching application 


\section{INTRODUCCIÓN}

En la actualidad existe una gran variedad de herramientas para el diseño y análisis de sistemas fotovoltaicos (Markvart y Castañer, 2003, Mellit et al., 2007). En el mercado se pueden encontrar desde simples herramientas de dimensionado, a otras más sofisticadas orientadas a la optimización y diagnóstico de estos sistemas. El grado de complejidad de cada una de estas herramientas, así como la exactitud en los resultados que permiten obtener, depende en gran medida de la función específica para la que han sido desarrolladas. En la enseñanza de los sistemas fotovoltaicos resulta de mucha utilidad el uso de este tipo de herramientas informáticas de modelado y simulación. La mayoría de ingenieros eléctricos y electrónicos están familiarizados con herramientas abiertas de programación y simulación, como Pspice (Cadence,1993) o Matlab (Mathworks, 1984), que pueden resultar de mucha utilidad en el modelado y simulación de sistemas fotovoltaicos.

Pspice es uno de los programas de mayor difusión para la simulación de circuitos electrónicos. Dadas sus capacidades y su popularidad entre los técnicos eléctricos y electrónicos, ha sido utilizado con éxito para el desarrollo de modelos de los distintos elementos que forman parte de un sistema fotovoltaico (Moreno et al., 2000 ; Silvestre et al., 1998; Castañer y Silvestre , 2002). A partir de los modelos desarrollados es posible un estudio detallado de las características y del funcionamiento de los sistemas fotovoltaicos en el dominio del tiempo. Las características de la simulación en términos de velocidad, posibilidades de tipos de análisis, entorno gráfico, y flexibilidad, son claras ventajas que aporta esta herramienta. Esta herramienta ha sido utilizada con éxito en la asignatura: Energía solar fotovoltaica, impartida en la titulación de Ingeniería de Telecomunicación de la Escuela Técnica Superior de Ingenieros de Telecomunicación de Barcelona, E.T.S.E.T.B., de la Universidad Politécnica de Cataluña, UPC (Silvestre et al., 1998, Castañer y Silvestre, 2002, E.T.S.E.T.B.-UPC, 2007).

Matlab (Mathworks, 1984), es otro de los entornos abiertos de cálculo numérico que ofrece avanzadas herramientas de manipulación matemática con un potente e intuitivo lenguaje de programación. Junto con Simulink, su entorno gráfico, se tiene una aplicación que logra un fácil manejo de las herramientas de simulación. Mathworks proporciona numerosos paquetes de librerías, toolboxes, para ampliar las prestaciones de análisis en múltiples campos de matemática aplicada. También incorpora interfaces con tarjetas de adquisición y control para enlazar el núcleo de cálculo con sistemas externos. Así como un enlace hacia Internet mediante un servidor Web. Pese a que las rutinas son potentes y variadas, el desarrollo de simulaciones de sistemas complejos no es trivial. Si bien el entorno Simulink es fácil de utilizar, la plataforma asume que el sistema será modelado a partir de ecuaciones diferenciales. Resulta necesario, pues, tener experiencia en la formulación de las ecuaciones y un profundo conocimiento de los sistemas a simular para conseguir una buena respuesta de los métodos de cálculo numérico. El uso de esta herramienta está cada día más extendido en ámbitos de ingeniería debido a la gran potencia y flexibilidad que ofrece. Por ese motivo se han desarrollado nuevos modelos, de los distintos elementos presentes en un sistema fotovoltaico, en entorno el Matlab/Simulink (Guasch et al., 2003, Silvestre et al., 2004). En este trabajo se presenta la aplicación académica de la utilización de Matlab/Simulink para la simulación de sistemas fotovoltaicos.

\section{MODELOS DESARROLLADOS EN MATLABISIMULINK}

Han sido desarrolladas librerías con los modelos de los principales elementos que se pueden incluir en un sistema de conversión fotovoltaica y que se detallan a continuación (Guasch y Silvestre, 2003, Silvestre et al., 2004): i) Baterías; ii) Módulos Fotovoltaicos; iii) Cargas DC y AC; iv) Reguladores de Carga; v) Inversores; vi) Convertidores DC/DC; vii) Puntos de conexión a Red; viii) Pérdidas del sistema

Los tipos de sistemas fotovoltaicos que se han incluido en las simulaciones en entorno Matlab/simulink, son los siguientes: a)

a) Sistema Flotante. Módulos fotovoltaicos con conexión directa a cargas DC.

b) Sistema Autónomo. formado por los módulos fotovoltaicos, baterías y cargas DC. 
c) Sistema Autónomo incluyendo pérdidas. El sistema autónomo con pérdidas intenta introducir el efecto del cableado en el modelo autónomo. Estas pérdidas pueden llegar a ser importantes por causas tan diversas como la longitud de los cables, las elevadas corrientes debidas a la baja tensión de trabajo, o a contactos deteriorados por envejecimiento y desgaste. Permiten, pues, modelar los efectos de caídas de tensión entre dispositivos y las fugas de corriente.

d) Sistema con Regulador de Batería. La utilización de un regulador de batería, o regulador de carga, permite incorporar las protecciones reales presentes en la mayoría de sistemas autónomos.

e) Sistemas Autónomos con salida $D C$ y AC. En estos sistemas, además de los elementos presentes en el punto anterior, se incluye un convertidor DC/AC o inversor y las correspondientes cargas AC.

f) Sistemas conectados a Red. En estos sistemas se incluye el generador fotovoltaico, un inversor y el punto de conexión a la red de distribución eléctrica. Se contempla también la posibilidad de que el inversor incluya seguimiento del punto de máxima potencia, así como la existencia de cargas AC.

En todos los casos, los modelos teóricos desarrollados han sido contrastados con medidas experimentales, evaluándose favorablemente las prestaciones del entorno de simulación. Se ha utilizado un banco de medidas empíricas como parámetros de entrada a las simulaciones constituido por datos de irradiancia, y temperatura. Las corrientes y tensiones relevantes de cada sistema han sido monitorizadas, en el Laboratorio de Sistemas Fotovoltaicos del Departamento de Ingeniería Electrónica de la UPC para ser correlacionados con los resultados de las simulaciones.

\section{EJEMPLO DE SIMULACIÓN}

Para ilustrar el potencial del procedimiento de simulación, se presenta un ejemplo mostrando la evolución detallada de las principales tensiones y corrientes en un sistema conectado a red. En este sistema, tanto el generador fotovoltaico, como las cargas AC presentes, están conectados a la red de distribución eléctrica. El generador dispone de un bloque DC/DC que realiza seguimiento del punto de máxima potencia del generador fotovoltaico y un inversor. Las cargas AC están directamente conectadas a la salida del inversor, como puede apreciarse en el diagrama de bloques de la figura 1. El generador fotovoltaico de la figura 1 consta de paneles solares Atersa A85, formados por 36 células en serie, Isc $=5.2 \mathrm{~A}, \mathrm{Voc}=21.2 \mathrm{~V}$, en una agrupación de 2 paneles en serie y 4 en paralelo. El inversor considerado es de $1 \mathrm{KWp}$ y las cargas $A C$ se componen de un conjunto de cargas resistivas de $120 \mathrm{~W}$ con un perfil de consumo predefinido.

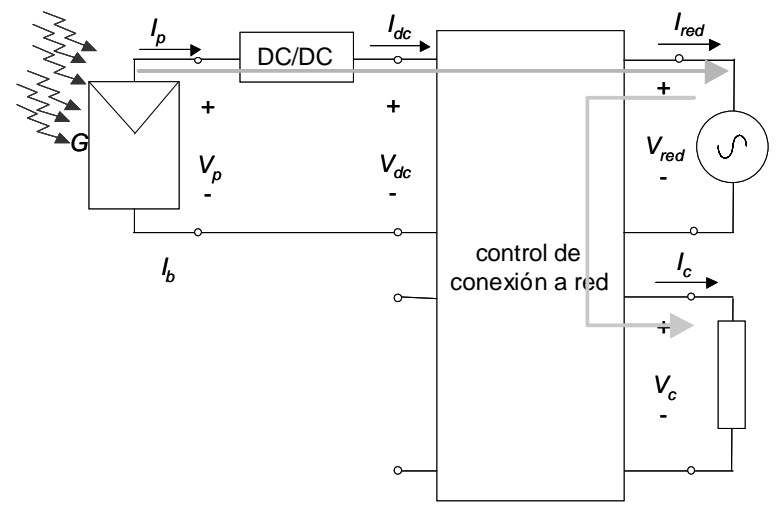

Fig. 1: Diagrama de bloques del Sistema. 


\section{Silvestre}

La figura 2 muestra la evolución de la tensión en la red eléctrica externa. Se ha introducido una caída de la red, alrededor de las 72 horas, para observar el comportamiento del sistema frente a una situación de islanding. Los parámetros meteorológicos de entrada, irradiancia y temperatura, son datos reales monitorizados a lo largo de 6 días en Barcelona, utilizándose un periodo de muestreo de 2 minutos, es decir, 4320 muestras para cada magnitud sensada. Las figuras 3 y 4 muestran, la evolución de la tensión y la corriente a la salida del generador fotovoltaico.

Como puede apreciarse en la figura 3, el generador fotovoltaico trabaja en el punto de máxima potencia, excepto en la franja temporal asociada a la caída de la red. Durante ese periodo de desconexión, los paneles quedan en circuito abierto, tal como se aprecia en la figuras 3 y 4 . El comportamiento de las cargas se muestra en la figura 5. La corriente sigue el perfil de consumo predefinido excepto en el intervalo de desconexión de la red. Finalmente, la figura 6 ilustra la evolución de la corriente en la red eléctrica, absorbiendo la corriente generada por los paneles y generando la corriente necesaria para las cargas.

El ejemplo presentado ha sido utilizado en la asignatura de Sistemas Fotovoltaicos del Master de Ingeniería Electrónica impartido en la Escuela Técnica Superior de Ingenieros de Telecomunicación de Barcelona de la UPC. Los estudiantes realizan 7 sesiones de laboratorio, de dos horas cada una, a lo largo del curso. Las prácticas están enfocadas al estudio de los sistemas fotovoltaicos a partir de simulaciones realizadas en entorno Matlab/Simulink. El acceso directo a los modelos utilizados en las simulaciones permite a los estudiantes una mejor compresión de los conocimientos impartidos sobre sistemas fotovoltaicos y una mejor comprensión de su funcionamiento.

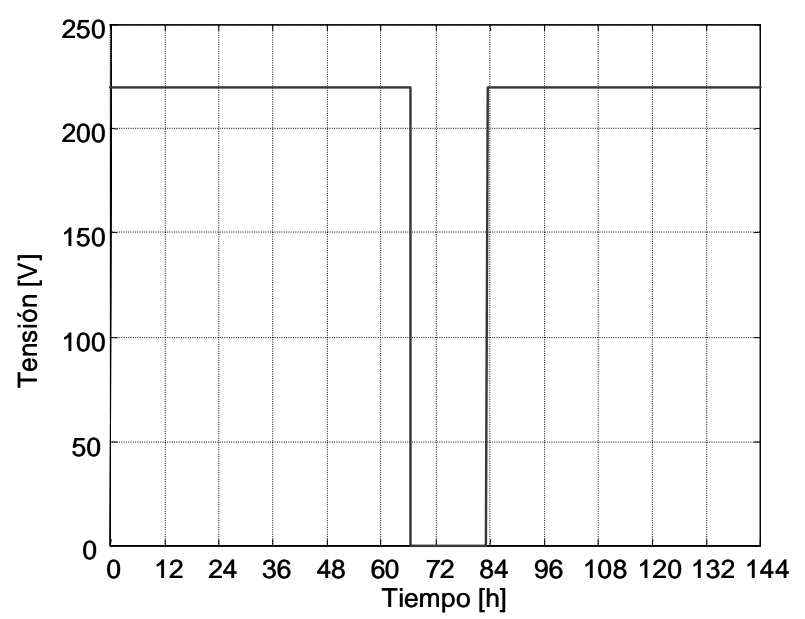

Fig. 2: Evolución de la tensión AC a la salida del inversor.

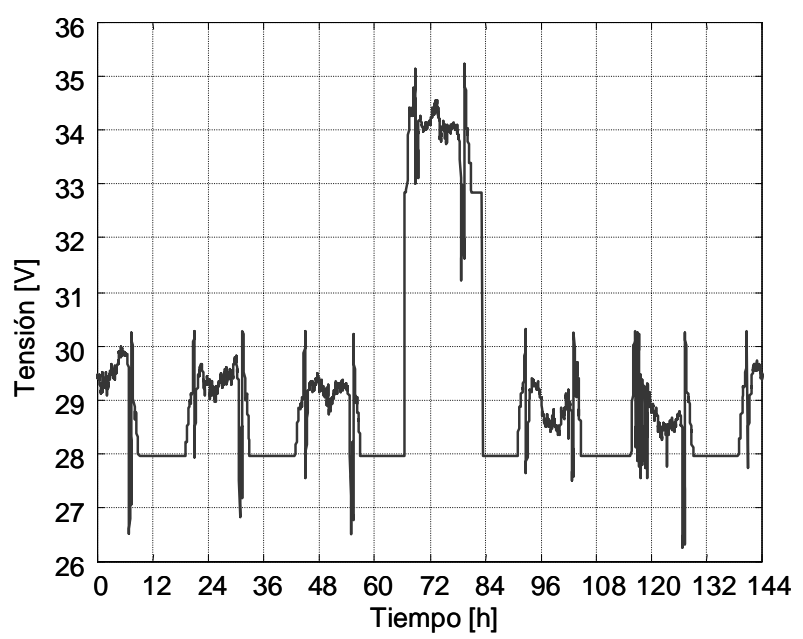

Fig. 3: Evolución de la tensión de salida del generador fotovoltaico. 


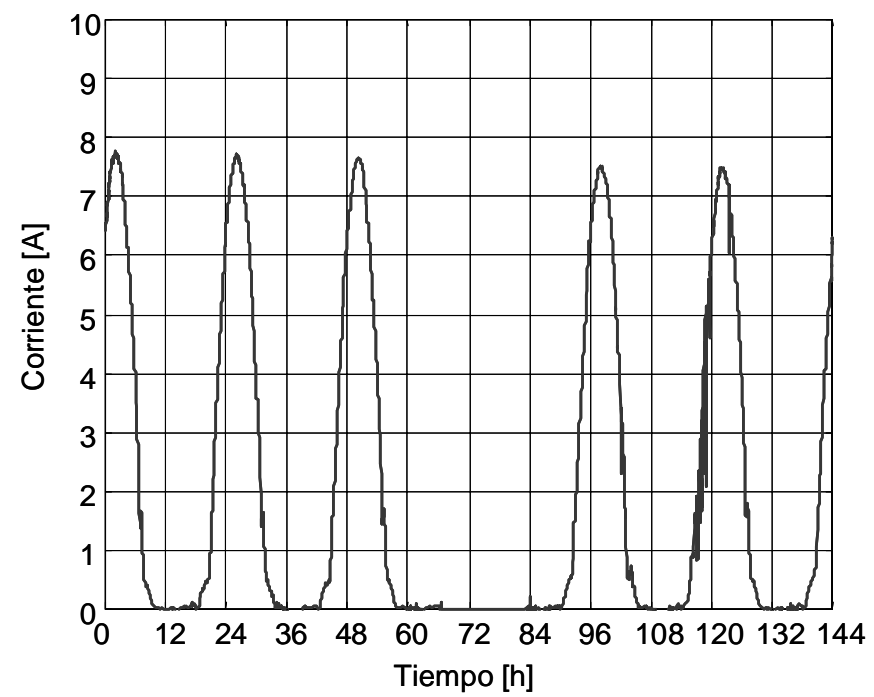

Fig. 4: Corriente a la salida del generador fotovoltaico.

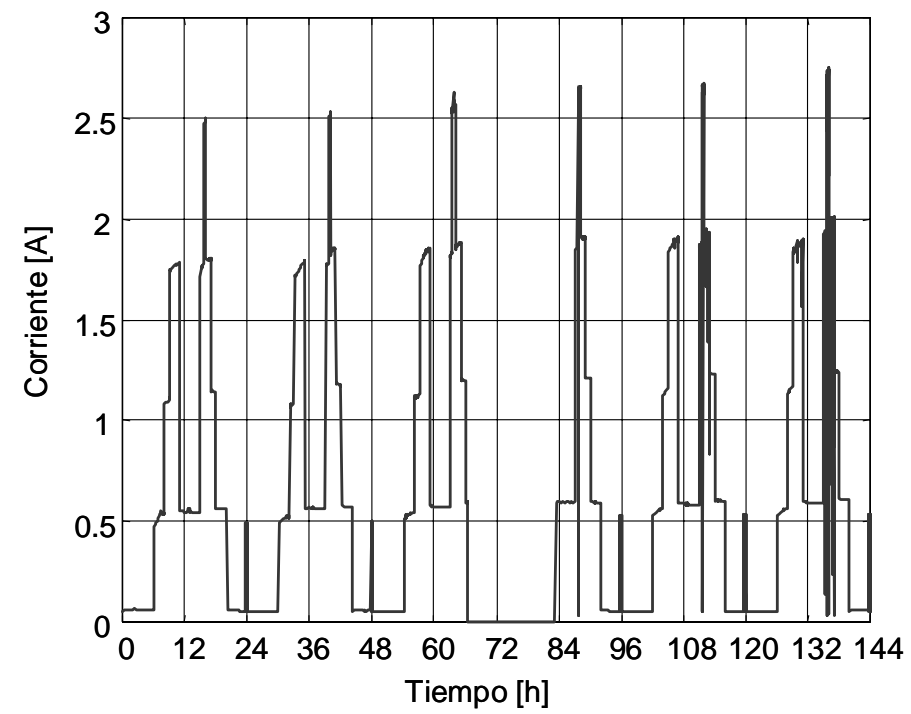

Fig. 5: Corriente en las cargas del sistema.

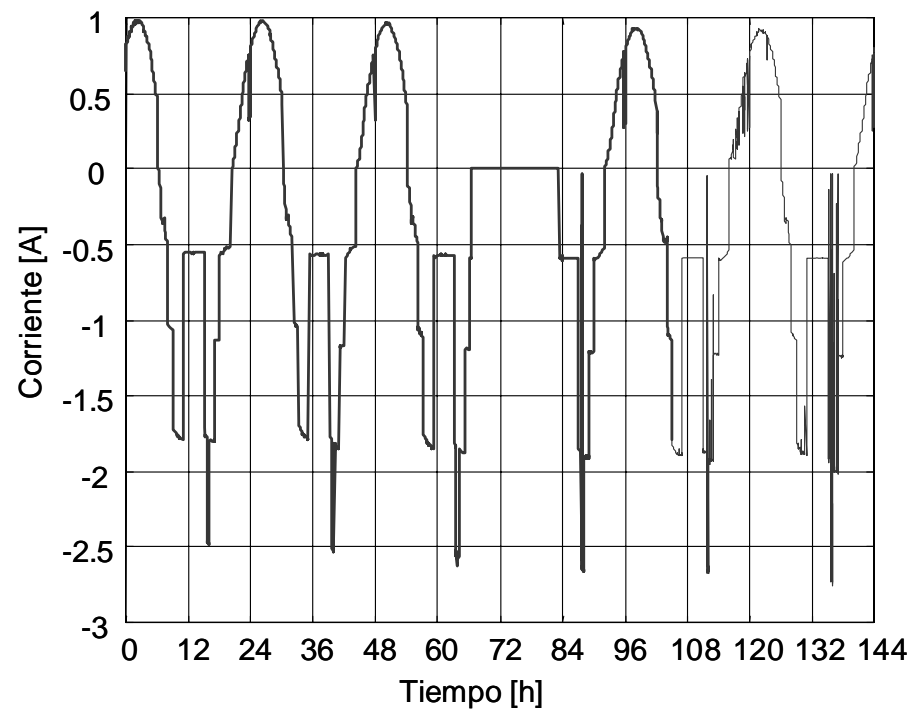

Fig. 6: Corriente inyectada o consumida de la red. 


\section{Silvestre}

Los estudiantes pueden modificar todos los parámetros de cada uno de los modelos desarrollados y ver así como afectan al funcionamiento y a las características del sistema en estudio. Además de las prácticas de laboratorio, a lo largo del curso se proponen a los estudiantes distintos tipos de actividades dirigidas, entre las que se encuentran: El estudio de distintos tipos de sistemas fotovoltaicos, realización de propuestas de dimensionado y análisis mediante simulación de su comportamiento bajo distintas condiciones de trabajo. En estos estudios se utilizan otras herramien-tas comerciales de simulación de sistemas fotovoltaicos como PVSOL (PVSOL, 2003) y PVSYST (PVSYST, 1996), que resultan de ayuda en el estudio de este tipo de sistemas, pero que no permiten el acceso a los modelos utilizados en las simulaciones, resultando por tanto menos flexibles y transparentes que las herramientas desarrolladas en entornos abiertos de programación.

\section{CONCLUSIONES}

El método de simulación de un sistema fotovoltaico basado en el entorno abierto de programación Matlab/Simulink propuesto en este trabajo permite un rápido y fácil acceso a las variaciones temporales de las corrientes y tensiones más importantes en los nodos del sistema. Las características de la simulación en términos de velocidad, tipos de análisis, entorno gráfico, flexibilidad para analizar distintas topologías y robustez, son ventajas claras de la potencia y funcionalidad de esta herramienta. Con la aplicación del ejemplo de simulación se permite a los alumnos, un acceso cómodo y rápido al análisis y dimensionado de un sistema fotovoltaico, así como la posibilidad de una gran potencia de cálculo en distintas condiciones de trabajo, lo que repercute en una visión global clara del sistema completo y de su comportamiento.

\section{REFERENCIAS}

Cadence (1993); Página web del distribuidor de la herramienta: http://www.cadence.com/orcad/ index.html, Acceso: 14 Septiembre 2007

Castañer L. y S. Silvestre; Modelling Photovoltaic Systems using Pspice, Wiley, Chichester, England (2002).

ETSETB-UPC; Página Web de la Escuela Técnica Superior de Ingenieros de Telecomunicación de Barcelona, UPC: www.etsetb.upc.es Acceso: Septiembre 2007.

Guasch D. y S. Silvestre; "Dynamic battery model for Photovoltaic applications", Progress in Photovoltaics: Research and applications: 11, 193-206 (2003).

Markvart T. y L. Castañer; "Practical Handbook of Photovoltaics: Fundamentals and Applications", pp.543-564, Oxford, UK, Elsevier (2003).

Mathworks (1984); Página web del distribuidor de la herramienta: http://www.mathworks.com/ Acceso: 15 Septiembre 2007.

Mellit A., M. Benghanem y S.A. Kalogirou; "Modeling and simulation of a stand-alone photovoltaic system using an adaptive artificial neural network: Proposition for a new sizing procedure", Renewable energy: 32(2), 285-313 (2007).

Moreno A., J. Julve, S. Silvestre y L. Castañer; "SPICE Macromodeling of Photovoltaic Systems", Progress in Photovoltaics: Research and Applications: 8(3), 293-306 (2000).

PVSOL (2003); Página Web del distribuidor del software PVSOL: http://www.valentin.de/ index_ es_page=pvsol Acceso : septiembre 2007.

PVSYST (1996); Página Web del distribuidor del software PVSYST; http://www.pvsyst.com/ Acceso : Septiembre 2007.

Silvestre S., L. Castañer, R. Aloy y D. Carles; Aplicación del Simulador Spice a la enseñanza de los sistemas fotovoltaicos. Información Tecnológica: 9(4), 345-348 (1998).

Silvestre S., D. Guasch, P. Ortega y R. Calatayud; "Photovoltaic Systems Modelling using Matlab and Simulink" Proc. of the 19th European Photovoltaic Solar Energy Conference, 19th PVSEC pp.2207-2210, Paris, France (2004). 